\title{
A Method of Signal Timing Optimization for Spillover Dissipation in Urban Street Networks
}

\author{
Dongfang Ma, ${ }^{1}$ Dianhai Wang, ${ }^{1}$ Yiming Bie, ${ }^{2}$ and Sun $\mathrm{Di}^{3}$ \\ ${ }^{1}$ College of Civil Engineering and Architecture, Zhejiang University, Hangzhou 310058, China \\ ${ }^{2}$ School of Transportation Science and Engineering, Harbin Institute of Technology, Harbin 150091, China \\ ${ }^{3}$ College of Automation, Beijing Union University, Beijing 100101, China
}

Correspondence should be addressed to Dianhai Wang; wangdianhai@zju.edu.cn

Received 2 November 2012; Accepted 3 February 2013

Academic Editor: Wudhichai Assawinchaichote

Copyright (C) 2013 Dongfang Ma et al. This is an open access article distributed under the Creative Commons Attribution License, which permits unrestricted use, distribution, and reproduction in any medium, provided the original work is properly cited.

\begin{abstract}
The precise identification and quick dissipation of spillovers are critically important in a traffic control system, especially when heavy congestion occurs. This paper first presents a calculation method for the occupancy per cycle under different traffic conditions and identifies the threshold of occupancy that characterizes the formation of spillovers. Then, capacity adjustments are determined for the incoming and outgoing streams of bottleneck links, with the aim of dissipating the queue to a permissible length within a given period of time, and optimization schemes are defined to calculate splits for the upstream and downstream intersections. Finally, taking average vehicular delay, outputs per cycle, and maximum queue length on the bottleneck link as the evaluation indices, the method of dissipating spillovers proposed in this paper is evaluated using a VISSIM simulation. The results show that the maximum queue length on the bottleneck link and the average vehicular delay at the upstream and downstream intersections decrease significantly under the new signal control plan; meanwhile, the new control schemes have little influence on the outputs of the two intersections per cycle.
\end{abstract}

\section{Introduction}

Spillovers occur when growing queues at the downstream signal block arrivals from the upstream signal, so that vehicles cannot depart even though the signal phase is green; spillovers may also appear when turning vehicles fill up the available storage length of turning bays and block through movements [1]. When the physical queues exceed the link length, vehicles mobility and pedestrians' safety at the upstream intersection may be restricted $[2,3]$.

It is already known that spillovers are the result of oversaturated conditions and can lead to an intensely increasing amount of queued traffic and more congestion in urban street networks. As the operation efficiency of upstream traffic signals, and even the entire network, can decrease dramatically under the influence of spillovers, identifying the conditions under which spillovers occur and dissipating the resulting queues is important. Such analysis can provide a basis for a traffic control plan, both in complex urban systems and at intersections.
Over recent decades, a significant number of studies have been devoted to modeling spillovers. Chang and Bertoli [4] derived a model that determined when a spillover blockage should be controlled, under the hypothesis that arrivals from upstream obey a uniform distribution. Wu et al. [5, 6] discussed the concepts of the two types of queue-overdetector (QOD), which are caused by spillovers or red signal phases, respectively, and presented a method of measuring the duration of the first type of QOD: a spillover can be said to have occurred if the value of the first type of QOD is greater than 0 . In addition, when spillovers from downstream block vehicles trying to depart from the upstream signal line, the queue will discharge at a lower rate than the saturation flow. Geroliminis and Skabardonis described the relationship between growing queues and high values of occupancy and determined the threshold values of occupancy at which spillovers occur $[7,8]$.

The method to identify spillovers in [4] is relatively complex, and it is developed with the assumption that the residual queue length at the beginning of red signal phase 
is known. Meanwhile, the three break points in $[5,6]$ which indicate a change in traffic state are difficult to identify when the bus ratio in the traffic flow is high. Although this method in $[7,8]$ is easier to realize, it neglects the effects of residual queue length from past cycles, which will induce marked influence on the threshold of occupancy with the increasing of the distance from the stop line to the detector.

As the negative influence of spillovers has become more and more serious in urban areas, and since the existing algorithms for signal coordination do not handle the temporal and spatial propagation of queues, the avoidance and dissipation of spillovers has attracted the interest of many researchers. To date, a large number of methods have been proposed to solve the spillover problem. Several, based on optimizing traffic signal parameters, are regarded as bottleneck controls. As a special traffic control mode, the objective function of a bottleneck control method is based on queue management and deviates from the traditional delay minimization concept [9]. The first model of queue management at an isolated intersection was invented by Gazis [10], for the context of one-way streets. Later, Girianna and Benekohal extended the method in [10] to two-way arterial networks [11,12]. Moreover, due to the complexity of these models, intelligent algorithms, such as genetic algorithms and Petri nets [13-15], were applied to optimize signal timing for spillovers, with the objective of minimizing the time spent in a queue.

The abovementioned measures of signal timing optimization for bottleneck controls are difficult to apply in practice, however, as there are some differences between the assumptions used in the papers and actual traffic conditions. Consequently, a recent report suggested that "no current generally available tool is adequate for optimizing signal timing in congested conditions, particularly at the intersection level" [16]. To close these gaps, this paper introduces a method of identifying spillovers based on the research results of [7] and develops a new method of signal timing optimization for the upstream and downstream intersections so as to dissipate spillovers. The remainder of this paper is organized as follows: the first part describes the methodology for spillover identification in a city street network with signalized intersections. Next, in Section 2, a method of signal time optimization is developed to control bottlenecks. Section 3 analyzes the efficiency of the new control measure proposed in this paper using a VISSIM simulation, taking the outputs of the downstream and upstream intersections, average vehicular delay across all links, and the queue length at the bottleneck link as the evaluation indices.

\section{Identification of a Spillover}

Fixed detectors are placed sufficiently far upstream from the intersection stop line (usually about 30 50 m), in SCAT (Sydney Coordinated Adaptive Traffic System), RHODES (Real-time, Hierarchical, Distributed, and Effective System), and other systems $[17,18]$. Traffic data, including the number of vehicles and the occupancy (the proportion of time that the detector is occupied), is obtained from the detectors, which provides a foundation for signal timing optimization.

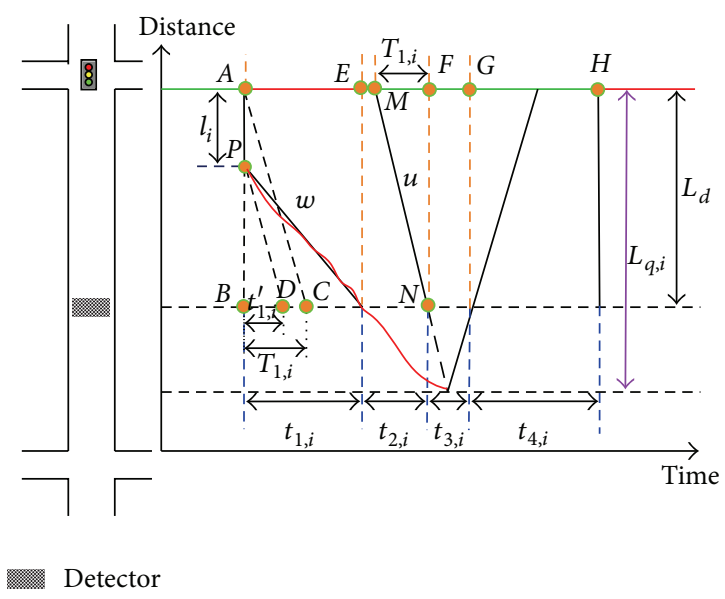

FIGURE 1: Shockwaves at an isolated signal.

2.1. Estimation of Occupancy Based on Fixed Traffic Data. Generally, the shockwaves at an isolated signal within one cycle can be depicted as in Figure 1, and the cycle time can be divided into four time intervals by using the detector position as a reference space point if the queues extend to the detector location.

Here, point $A$ is the beginning of cycle $i \#$, and it is also the starting point of the red light for this phase during cycle $i \# ; E$ and $F$ are the times at which the stopping and starting waves approach the detector, respectively; $M$ is the beginning of the green phase of cycle $i \# ;$ is the time at which the queue length is equal to $L_{d}$, after dissipating for a certain time period; $H$ is the end of the green phase of cycle $i \#$; point $C$ represents the time at which the stopping wave reaches the detector location, ignoring the residual queue at the beginning of the red phase and assuming that the traffic arrivals approach the saturation flow rate; finally, point $C$ should be replaced by $D$ if the residual queue is $l_{i}$.

In Figure $1, t_{2, i}$ is the time interval over which the measured vehicle count of the fixed detector is 0 , that is, the difference between $E$ and $F$; $t_{1, i}$ is the length of time over which the queues do not yet extend to the position of the detector, while vehicles are arriving during the red phase, and is equal to the difference between $A$ and $E ; t_{3, i}$ is the length of time for which the queues discharge at the saturation flow; $t_{4, i}$ is the remaining green signal time during which platoons cross the stop line with no interference from the traffic signal, and $T_{1, i}$ is the time it takes for the starting wave to reach the location of the detector from the stopping line, that is, the difference between $M$ and $F . L_{d}$ is the distance between the detector and the stop line, and $L_{q, i}$ is the maximum queue length in cycle $i \#$.

As discussed in [7], when the growing queues extend as far as the detector $\left(t_{2, i}>0\right)$, the platoon arrivals are not known during the time period $t_{2, i}$, and the occupancy for cycle $i \#, o_{i}$, can be calculated piecewise, as given by

$$
o_{i}=\frac{\sum_{j=1,3,4} t_{j, i}\left(L_{\mathrm{eff}} q_{j, i} / u_{f}\right)+t_{2, i} \cdot o_{\text {stop }}}{c},
$$


where $c$ is the cycle time (s); $L_{\text {eff }}$ is the speed estimation parameter in units of distance known as the average effective vehicle lengths of the traffic stream $(\mathrm{m}) ; u_{f}$ is the free-flow speed $(\mathrm{m} / \mathrm{s})$, usually regarded as fixed; $t_{j, i}$ is the time for interval $j$ in cycle $i \#(s) ; q_{j, i}$ is vehicle count during period $t_{j, i}$ (veh).

The first term is the free-flow occupancy, and the second expresses the congested conditions at the detector. The term $o_{\text {stop }}$ is the jam occupancy, with a value close to 1 , and $q_{j, i}$ satisfies $\sum_{j=1,3,4} t_{j, i} \cdot q_{j, i}=\bar{q}_{i} \cdot c$. Here, $\bar{q}_{i}$ is the average flow measured by the detector in interval $i$ (veh/s).

The free-flow speed data can be obtained from the data under unsaturated conditions, and the effective vehicle length of the traffic stream is approximately equal to the average vehicle length plus the detector length [15]. Then rewriting (1), we have

$$
o_{i}=\frac{\bar{q}_{i} \cdot L_{\mathrm{eff}}}{u_{f}}+\frac{t_{2, i} \cdot o_{\text {stop }}}{c} .
$$

The estimation of $t_{2, i}$, which is based only on values of observable quantities, is an important parameter for estimating $o_{i}$ and is the basis of analyzing the threshold of occupancy at which spillovers appear.

If the starting wave intersects with the stopping wave in front of the detector, $t_{1, i}, T_{1, i}$ and $r$ must satisfy $r+T_{1, i}<$ $t_{1, i}$, and the value of $t_{2, i}$ must be 0 (as shown in Figure 1); meanwhile, if the queues can extend as far as the detector in cycle $i \#, r+T_{1, i}>t_{1, i}$ is tenable, and $t_{2, i}$ must be equal to $r+T_{1, i}-t_{1, i}$. Accordingly, a general form of $t_{2, i}$ is as follows:

$$
t_{2, i}=\max \left\{0, r+T_{1, i}-t_{1, i}\right\} .
$$

Here, $r$ is the red phase time (s).

$T_{1, i}$ should not be greater than the green phase time, $g$, that is, $T_{1, i}<g$. However, if the starting wave cannot extend as far as the detector during the green phase of one cycle, $T_{1, i}>g$ is tenable, and $t_{2, i}$ should equal $c-t_{1, i}$ in this situation. Then, (3) should be modified as

$$
t_{2, i}=\max \left\{0, \min \left\{r+T_{1, i}-t_{1, i}, c-t_{1, i}\right\}\right\} .
$$

With the traffic signal parameters known, the value of $t_{2, i}$ is determined by $T_{1, i}$ and $t_{1, i}$, which can be obtained using the stopping and starting wave models.

2.2. Threshold of Occupancy That Leads to Spillover. When the residual queue is less than $L_{d}, t_{1, i}$, the time when the stopping wave reaches the detector, is influenced by the traffic flow upstream and the residual queue at the beginning of the red phase, and the corresponding value is at a minimum if the traffic flow is equal to the saturated flow (i.e., the stopping and starting waves have the same speeds). If $P$ is the farthest point in the queue at the beginning of the red phase, we can produce the following formula by analyzing the relationships between $A, B$, and $C$ in Figure 1 :

$$
\frac{t_{1, i}^{\prime}}{T_{1, i}}=\frac{L_{d}-l_{i}}{L_{d}},
$$

where $t_{1, i}^{\prime}$ is the minimum value of $t_{1, i}$ (s) over cycle $i$, and $l_{i}$ is the residual queue at the beginning of the red phase of cycle $i$ (m).

Rewriting (5), we obtain

$$
t_{1, i}^{\prime}=\left(1-\frac{l_{i}}{L_{d}}\right) T_{1, i} .
$$

If $l_{i}$ is less than $L_{d}$, then the value of $t_{1, i}^{\prime}$ can range from 0 to $T_{1, i}$, while $t_{2, i} \leq \min \left\{T_{1, i}+r, c\right\}$.

If $l_{i}$ is no less than $L_{d}$ at the beginning of the red phase in cycle $i, t_{1, i}=0$ and $t_{2, i}=\min \left\{T_{1, i}+r, c\right\}$ are both tenable. Therefore, $t_{2, i}$ under the influence of the residual queue length must satisfy

$$
t_{2, i} \leq \min \left\{r+T_{1, i}, c\right\}
$$

$T_{1, i}$ can be estimated from the starting wave speed and $L_{d}$ according to traffic wave theory, which gives

$$
T_{1, i}=\frac{L_{d}}{u_{w}}
$$

Here, $u_{w}$ is the starting wave speed $(\mathrm{m} / \mathrm{s})$.

Substituting (8) into (7), we have

$$
t_{2, i} \leq \min \left\{r+\frac{L_{d}}{u_{w}}, c\right\} .
$$

Meanwhile, substituting (9) into (2) gives the occupancy during cycle $i$ without taking the effect of spillovers into consideration:

$$
o_{i} \leq \frac{\bar{q}_{i} \cdot L_{\text {eff }}}{u_{f}}+\min \left\{\frac{r}{c}+\frac{L_{d}}{c \cdot u_{w}}, 1\right\} .
$$

The critical value for the blocking occupancy per cycle, that is, the maximum occupancy that is possible without causing spillovers, is given by

$$
o_{i, \max }=\frac{\bar{q}_{i} \cdot L_{\mathrm{eff}}}{u_{f}}+\min \left\{\frac{r}{c}+\frac{L_{d}}{u_{w} \cdot c}, 1\right\} .
$$

Thus, if the measured occupancy in cycle $i$ is greater than the critical value $o_{i \text { max }}$, we can conclude that spillovers from the downstream link have blocked departures from the first link upstream, and that new traffic control strategies need to be put in place at the upstream and downstream intersections.

\section{Optimization of Signal Timing}

Signal timing plans are determined by many factors, which can be divided into two classes: the traffic flow of different streams and the geometric layouts of intersections. Taking Figure 2 as an illustration, the subarea contains a set $L=$ $\left\{L_{n} \mid n=1,2, \ldots N\right\}$ of $N$ links where each link represents a road between two subsequent intersections. All links except $L_{i+1,3}$ can be classified as either (1) input links for $L_{i+1,3}$, pertaining to the set $L_{\text {in }} \subset L$, that are controlled by a traffic light located at the upstream intersection and include $L_{i, 1}$, 


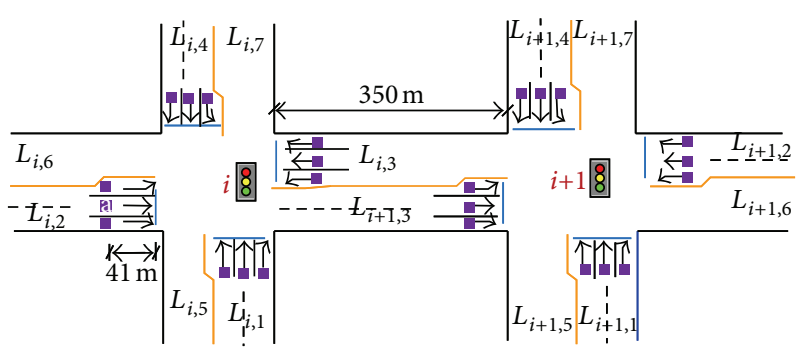

Detector

FIGURE 2: Experimental region of simulation.

$L_{i, 2}, L_{i, 4}$, (2) output links for $L_{i+1,3}$, pertaining to the set $L_{\text {out }} \subset L$, that are controlled by a traffic light located at the downstream intersection and include $L_{i+1,5}, L_{i+1,6}, L_{i+1,7}$, or (3) unconnected links pertaining to the set $L_{\text {int }} \subset L$, that have no direct connection with $L_{i+1,3}$. For a generic link $L_{n} \in L$ with $n=1,2, \ldots N$, the sets $L_{\text {in }}^{n}$ and $L_{\text {out }}^{n}$ represent, respectively, the sets of incoming and outgoing links for $L_{n}$.

Here, $i+1$ is the downstream intersection, and $L_{i+1,3}$ is the link of interest, where spillovers occur frequently. In this paper, we assume that every approach has three lanes, whose attributes are through, left turning, and right turning, respectively. Moreover, the traffic signal plans for the two intersections both use a four-phase model, and we assign 1, 2, 3, and 4 to represent the East-West through, E-W left turn, South-North through, and S-N left turn phases, respectively.

\subsection{Capacity Adjustments for Inputting and Outputting} Streams. As shown in Figure 2, the inputs to the bottleneck link consist of three parts: the through stream on $L_{i, 2}$, the left-turn stream on $L_{i, 4}$, and the right-turn stream on $L_{i, 1}$; meanwhile, the outputs per cycle are restricted by the capacities of the three outputting streams on $L_{i+1,3}$. A spillover can dissipate only when the inputs to the bottleneck link are fewer than the outputs within a given unit of time.

Generally, other approaches (such as $L_{i, 1}, L_{i, 2}, L_{i, 3}, L_{i, 4}$, $\left.L_{i+1,1} L_{i+1,2}, L_{i+1,4}\right)$, including the bottleneck link, are operated with a high saturation degree when spillovers occur, and the bottleneck control should not exert an excessively negative influence upon them, or else new traffic congestion will appear. Thus, there should be a balance between the dissipation of the queue and the negative influence on other links; the optimization interval, $T^{*}$, should be not too small or too large, and can be set to a constant value in an actual application. If the vehicles on $L_{i+1,3}$ can exit freely; that is, the queues on $L_{i+1,5}, L_{i+1,6}$, and $L_{i+1,7}$ cannot block vehicles from departing when the signal phase at intersection $(i+1)$ is green, then the optimization interval and the traffic signal timing of the two intersections satisfies

$$
T^{*}=\frac{1}{l} \cdot \frac{L_{m, \max }-L_{m, \text { ideal }}}{\sum_{h \in L_{\text {out }}^{m}} \lambda_{m, h} \cdot Q_{m, h}-\sum_{z \in L_{\text {in }}^{m}} \min \left\{q_{z, m}, \lambda_{z, m} \cdot Q_{z, m}\right\}},
$$

where $T^{*}$ is the optimization interval for the bottleneck control, $m$ represents the bottleneck link, $L_{i+1,3}$ in this paper,
$L_{m, \max }$ is the maximum queue length in the bottleneck link at the beginning of the cycle when the bottleneck control is implemented, which should depend on the residual queue lengths in $L_{i+1,2}$ and $L_{i+1,4}, L_{m \text {,perm }}$ is the permissible queue length after applying the bottleneck control, $l$ is the average headway between any two sequential vehicles, approximately $7 \mathrm{~m}$ when the traffic condition is congested, $h$ is the link of the intersection $(i+1)$, where $h \in L_{\text {out }}^{m}, \lambda_{m, h}$ is the split of the phase which contains the stream from link $m$ to link $h, Q_{m, h}$ is the saturation flow rate of the stream from link $m$ to link $h$, $z$ is one link of the upper intersection, where $z \in L_{\text {in }}^{m}, \lambda_{z, m}$ is the split of the phase which contains the stream from link $z$ to link $m, Q_{z, m}$ is the saturation flow rate of the stream from link $z$ to link $m$ and $q_{z, m}$ is the traffic flow rate from link $z$ to $m$.

Both of the through and left-turn streams on the bottleneck link are congested during the period of the bottleneck control, and vehicles in the two streams will discharge at the saturated flow rate. If we neglect the difference between the inputs and the outputs on the bottleneck link under the former signal timing plans, then the outputs from link $m$ per cycle must be greater than the inputs from the upstream intersection per cycle during the period of the bottleneck control, under the premise of dissipating spillovers, and the difference per unit of time can be written as

$$
\Delta S_{a}=\frac{L_{m, \text { max }}-L_{m, \text { ideal }}}{T^{*} \cdot l} .
$$

There is a relationship between the through and turning movements on link $m$, and the number of vehicles passing through the downstream intersection since the left-turn stream per cycle cannot be greater than the storage capacity of the channelization area (as seen in Figure 1). Thus, the output capacity of link $m$ can be written as

$$
\begin{aligned}
Q_{\text {out }, m}= & \left\{\sum_{h \in L_{\text {out }}^{m} t h \neq L_{i+1,7}}\left(\lambda_{m, h} \cdot Q_{m, h}\right)\right. \\
& \left.+\sum_{h=L_{i+1,7}} \min \left(\lambda_{m, h} \cdot Q_{m, h}, \frac{L_{m, h}^{\prime}}{l \cdot c_{i+1}}\right)\right\},
\end{aligned}
$$

where $L_{m, h}^{\prime}$ is the channelization length of the left-turn lane in link $m$ and $c_{i+1}$ is the cycle length under the former signal plan for intersection $(i+1)$.

Then, the difference between the outputs and inputs per unit of time under the former control plan is

$$
\Delta S_{d}=Q_{\mathrm{out}, m}-\sum_{z \in L_{\mathrm{in}}^{m}} \min \left(q_{z, m}, \lambda_{z, m} \cdot Q_{z, m}\right)
$$

In order to reduce the queue length in the bottleneck to a permissible value within the optimization interval, we should increase the output capacity and/or decrease the input capacity of the bottleneck. Then, the total capacity adjustment for the input and output streams can be written as

$$
\Delta S=\Delta S_{a}+\Delta S_{d}
$$


In order to balance the inputs and outputs of the bottleneck link and avoid the appearance of a saturation unbalance among the different traffic flows, the total capacity adjustment, $\Delta S$, must be distributed by taking the input and output stream capacities under the former signal timing plan as the criterion. Assuming that the increase of the output stream capacities and the decrease of the input stream capacities for link $m$ are $\Delta S_{m+1}$ and $\Delta S_{m}$, respectively, then the capacity adjustments for the two streams are

$$
\begin{gathered}
\Delta S_{m}=\Delta S \frac{S_{m}}{S_{m}+S_{m+1}}, \\
\Delta S_{m+1}=\Delta S \frac{S_{m+1}}{S_{m}+S_{m+1}},
\end{gathered}
$$

where $S_{m}$ is the total capacity of the traffic streams from link $z$ to link $m$ under the former signal timing plan (veh/s), $z \in L_{\text {in }}^{m}$, and $S_{m+1}$ is the total capacity of the streams from link $m$ to link $h$ under the former plan (veh/s), $h \in L_{\text {out }}^{m}$.

3.2. Signal Timing for Downstream Intersection. The traffic state in other approaches to the downstream intersection will also be congested in the event of a spillover, and the cycle time of the intersection may have reached a maximum, indicating that the capacities of the output streams on the bottleneck link could be increased by simply adjusting the splits. Moreover, the right-turning stream can pass through the intersection freely without any influence from the signal control, so the capacity adjustments for different phases could be obtained by taking the through and left-turn movement capacities under the former signal plan as the weights, giving

$$
\Delta S_{m, h}=\frac{\lambda_{m, h} \cdot Q_{m, h}}{\sum_{h \in L_{\text {out }}^{m} \mid h \neq L_{i+1,5}} \lambda_{m, h} Q_{m, h}} \Delta S_{i+1},
$$

where $\Delta S_{m, h}$ is the capacity adjustment of the stream from link $m$ to link $h$ after the bottleneck control has been adopted, $h \in L_{\text {out }}^{m} \mid h \neq L_{i+1,5}$.

The ratio of $\Delta S_{m, h}$ to $Q_{m, h}$ is the ideal split adjustment for the stream from link $m$ to link $h$; however, some new spillovers will appear on other links if the corresponding splits of the other phases are decreased too much. In order to prevent new oversaturation conditions from appearing and avoid aggravating the existing oversaturation conditions on the other links, a maximum allowable reduction in the splits for the other streams must be set when determining $\Delta S_{m, h}$. Assuming that $J_{i+1}$ is the set of phases during which the vehicles on the bottleneck link must wait in a queue because of a red light, the minimum split for phase $j, j \in J_{i+1}$, is determined by the saturation threshold, that is,

$$
\lambda_{j, \min }=\frac{q_{j}}{\max \left(x_{j, \max }, x_{j}\right) \cdot Q_{j}} .
$$

Here, $\lambda_{j, \min }$ is the minimum split of phase $j$ given the saturation threshold, $q_{j}$ is the key traffic flow of phase $j, x_{j, \max }$ is the saturation threshold in phase $j$, generally taken to be 0.95 in actual applications, $x_{j}$ is the degree of saturation in phase $j$, and $Q_{j}$ is the saturated flow for the key traffic flow in phase $j$.

Let $\bar{J}_{i+1}$ represent the set of phases during which the through or left-turn stream can depart from link $m ; \bar{J}_{i+1}=$ $\{3,4\}$ in this paper. The key traffic flow in phase $p$ must be one of the streams from link $m$ to link $h$; otherwise, the spillover should have occurred on link $L_{i+1,2}$ instead. Now, (18) can be rewritten as

$$
\Delta S_{p}=\frac{\lambda_{p} \cdot Q_{p}}{\sum_{p \in \bar{J}_{i+1}} \lambda_{p} \cdot Q_{p}} \Delta S_{i+1},
$$

where $\lambda_{p}$ is the split of phase $p, p \in \bar{J}_{i+1}$, and $Q_{p}$ is the saturated flow of the key traffic flow for phase $p$.

The total split increment of phase $p, p \in \bar{J}_{i+1}, \Delta \lambda_{i+1}$, is

$$
\begin{aligned}
\Delta \lambda_{i+1}=\min & \left\{\sum_{p \in \bar{J}_{i+1}} \frac{\Delta S_{p}}{Q_{p}},\right. \\
& \left.\sum_{j \in J_{i+1}}\left(\lambda_{j}-\frac{q_{j}}{\max \left\{x_{j, \max }, x_{j}\right\} \cdot Q_{j}}\right)\right\} .
\end{aligned}
$$

Using the splits from the former plan as the weights, the total increase in the splits, $\Delta \lambda_{i+1}$, can be distributed approximately equally. Then, the adjustment of phase $p, p \in$ $\bar{J}_{i+1}$, is as follows:

$$
\Delta \lambda_{p, i+1}=\frac{\lambda_{p, i+1} \cdot \Delta \lambda_{i+1}}{\sum_{p \in \bar{J}_{i+1}} \lambda_{p, i+1}} .
$$

Here, $\Delta \lambda_{p, i+1}$ is the adjustment in phase $p, p \in \bar{J}_{i+1}$.

The actual split of phase $p, p \in \bar{J}_{i+1}$, can be written as

$$
\lambda_{p, i+1}^{\prime}=\lambda_{p, i+1}+\Delta \lambda_{p, i+1},
$$

where $\lambda_{p, i+1}^{\prime}$ is the actual split of phase $p$ during the bottleneck control.

As the total number of splits for all phases is constant, the number of splits in the S-N through and S-N left-turning phases must be reduced to match the increases in the splits of the E-W through and E-W left-turning phases. Taking the splits in the former traffic signal plan as the weights, the reduction in phase $j, j \in J_{i+1}$, is as follows:

$$
\Delta \lambda_{j, i+1} \frac{\lambda_{j, i+1} \cdot \Delta \lambda_{i+1}}{\sum_{j \in J_{i+1}} \lambda_{j, i+1}},
$$

Here, $\Delta \lambda_{j, i+1}$ is the adjustment to phase $j, j \in J_{i+1}$.

The actual split in phase $j$ during the bottleneck control, $j \in J_{i+1}$, is

$$
\lambda_{j, i+1}^{\prime}=\lambda_{j, i+1}-\Delta \lambda_{j, i+1},
$$

where $\lambda_{j, i+1}^{\prime}$ is the actual split in phase $j$ during the bottleneck control. 
3.3. Signal Timing for the Upstream Intersection. The cycle time of the upstream intersection should not be changed; otherwise, the coordinated control between it and its neighboring intersections will be destroyed. If the right-turning movement from $L_{i, 1}$ to $L_{i+1,3}$ can pass through the intersection $i$ freely, then the phases of the through movement on $L_{i, 2}$ and the left-turn movement on $L_{i, 4}$ should reduce the input capacity so as to dissipate the queue at the bottleneck. The ideal reduction in the two streams is $\Delta S_{i}$. If we distribute this decrease by taking the corresponding input capacities in the former traffic signal plan as the weights, then the split adjustment for the stream from link $z$ to link $m$ is

$$
\begin{aligned}
\Delta \lambda_{z, m}=\min \{ & \frac{\lambda_{z, m} \cdot Q_{z, m} \cdot \Delta S_{i}}{\sum_{z \in L_{\mathrm{in}}^{m}} \mid z \neq L_{i, 1} \lambda_{z, m} \cdot Q_{z, m}}, \\
& \left.\lambda_{z, m}-\frac{q_{z, m}}{\max \left\{x_{z, m, \max }, x_{z, m}\right\} \cdot S_{z, m}}\right\},
\end{aligned}
$$

where $\Delta \lambda_{z, m}$ is the split adjustment for the stream from link $z$ to link $m, z$ is one link of intersection $i$, where $z \in L_{\text {out }}^{m}$, $x_{z, m, \max }$ is the saturation threshold for the stream from link $z$ to link $m, \lambda_{z, m}$ is the spilt for the stream from link $z$ to link $m$ in the former signal timing plan, $Q_{z, m}$ is the saturated flow for the stream from link $z$ to link $m$, and $q_{z, m}$ is the traffic flow for the stream from link $z$ to link $m$.

The difference between $\lambda_{z, m}$ and $\Delta \lambda_{z, m}$ is the actual value of split for the stream from link $z$ to link $m$, which is

$$
\lambda_{z, m}^{\prime}=\lambda_{z, m}-\Delta \lambda_{z, m}
$$

where $\lambda_{z, m}^{\prime}$ is the actual split for the stream from link $z$ to link $m$ during the bottleneck control.

The total decrease in the split for the two input streams at intersection $i$ can be obtained from the following, taking the saturation threshold into account:

$$
\Delta \lambda_{i, \mathrm{dec}}=\sum_{z \in L_{\mathrm{in}}^{m} \mid z \neq L_{i, 1}} \lambda_{z, m}
$$

Let $J_{i}$ be the set of phases during which no vehicles in the queue can enter the bottleneck link, where $J_{i}=\{2,3\}$ in this paper; then, the split increscent for phase $j, j \in J_{i}$, given the maximum green phase time, is

$$
\Delta \lambda_{i, j}=\min \left\{\frac{\lambda_{i, j} \cdot \Delta \lambda_{i}}{\sum_{j \in J_{i}} \lambda_{i, j}}, \max \left\{0,\left(\frac{g_{i, j, \max }}{c_{i}}-\lambda_{i, j}\right)\right\}\right\} \text {. }
$$

Here, $\Delta \lambda_{i, j}$ is the split increment of phase $j$ at intersection $i, \Delta \lambda_{i, j}$ is the former split for phase $j$ at the upstream intersection, $g_{i, j, \max }$ is the maximum green time in phase $j$, and $c_{i}$ is the cycle length of the upstream intersection.

Similarly, the sum of $\lambda_{i, j}$ and $\Delta \lambda_{i, j}$ is the practical split for phase $j$ at intersection $i$ :

$$
\lambda_{i, j}^{\prime}=\lambda_{i, j}+\Delta \lambda_{i, j}
$$

TABLE 1: Link lengths at the two intersections.

\begin{tabular}{lcccc}
\hline \multirow{2}{*}{ Intersection } & \multicolumn{4}{c}{ Link length (m) } \\
& Approach 1 & Approach 2 & Approach 3 & Approach 4 \\
\hline$i$ & 550 & 580 & 350 & 550 \\
$i+1$ & 550 & 550 & 350 & 550 \\
\hline
\end{tabular}

The total of the split increments in phase $j$ at intersection $i, j \in J_{i}$, is

$$
\Delta \lambda_{i, \text { inc }}=\sum_{j \in J_{i}} \Delta \lambda_{i, j}
$$

Given the maximum green phase time and the saturation threshold, the total decrease in splits for the two input streams will be greater than the total increase in splits in the other phases; as a result, the green light interval must be prolonged by

$$
\Delta I_{i}=\max \left\{0, \frac{1}{2}\left(\Delta \lambda_{i, \mathrm{dec}}-\Delta \lambda_{i, \mathrm{inc}}\right) c_{i}\right\}
$$

\section{Technology Assessments}

Interference between the through and left-turning movements can be very serious, especially under heavy congestion, and may cause the vehicle discharge rate on the bottleneck link to be less than the saturated flow rate. Meanwhile, the residual queues on all approaches to the upstream intersection will affect the dissipation of spillovers. So, some deviations will occur from the optimized signal timing described above, and it is necessary to collect relevant traffic data so as to evaluate the new method.

4.1. Data Collection and Relevant Parameters. Traffic flow in an urban street network is clearly unrepeatable, and there are a large number of problems involved in evaluating the effects of a bottleneck control scheme on actual traffic data. Consequently, we collect traffic data using a VISSIM simulation under different conditions. The experimental region was shown in Figure 2. The distance between the fixed detector and the stop line at link $L_{i, 2}$ is about $41 \mathrm{~m}$, and the link lengths of the eight approaches to the two intersections are listed in Table 1 .

Meanwhile, the signal timings at the two intersections are presented in Table 2; the duration of the change interval between phases is $3 \mathrm{~s}$.

Generally, the amount of traffic will vary depending on the time of day, and spillovers may be more likely to appear during peak hours. In this paper, we hold the amount of traffic constant during each given interval, as listed in Table 3.

In actual applications in some cities in China, the ideal interval for dissipating a spillover is around $400 \mathrm{~s}$, and the permissible queue length after implementing the control on a bottleneck is about one half of the link length. The key parameters in VISSIM are set as follows: (1) the ratio of right-turning to through to left-turning vehicles on all approaches except $L_{i, 1}$ and $L_{i, 4}$ is $1: 2: 1$, and the corresponding ratios on $L_{i, 1}$ and $L_{i, 4}$ are $3: 3: 2$ and $1: 2: 2$, respectively; 
TABLE 2: Signal timing plans of the two intersections under the original scheme.

\begin{tabular}{lcccc}
\hline \multirow{2}{*}{ Intersection } & \multicolumn{4}{c}{ Green time (s) } \\
& Phase 1 & Phase 2 & Phase 3 & Phase 4 \\
\hline$i$ & 24 & 21 & 32 & 31 \\
$i+1$ & 32 & 20 & 36 & 20 \\
\hline
\end{tabular}

TABLE 3: Inputs to all approaches during each simulation hour.

\begin{tabular}{lccccc}
\hline Simulation hour & $0-1$ & $1-2$ & $2-3$ & $3-4$ & $4-5$ \\
\hline Traffic flow (veh/h) & 600 & 700 & 800 & 700 & 600 \\
\hline
\end{tabular}

TABLE 4: Signal timing plans of the two intersections under the bottleneck control.

\begin{tabular}{lcccc}
\hline \multirow{2}{*}{ Intersection } & \multicolumn{4}{c}{$\begin{array}{c}\text { Green time under the bottleneck } \\
\text { control scheme (s) }\end{array}$} \\
& Phase 1 & Phase 2 & Phase 3 & Phase 4 \\
\hline$i$ & 32 & 28 & 24 & 24 \\
$i+1$ & 24 & 15 & 44 & 25 \\
\hline
\end{tabular}

(2) the permissible queue length after the bottleneck control is $150 \mathrm{~m}$; (3) the bus ratio on all approaches is $5 \%$; (4) the expected speeds of cars and buses are $15 \mathrm{~m} / \mathrm{s}$ and $10 \mathrm{~m} / \mathrm{s}$ respectively; (5) the random seed is $41 ;(6)$ a queue is defined as beginning when the speed is less than $0.28 \mathrm{~m} / \mathrm{s}$, and ending when the speed is greater than $1.39 \mathrm{~m} / \mathrm{s}$.

Based on these parameters, the total capacity adjustment of the two intersections after a spillover has occurred, $\Delta S$, is approximately $818 \mathrm{veh} / \mathrm{h}$, as estimated by the method described in Section 2. The signal time parameters for the two intersections after the bottleneck control has been implemented are listed in Table 4 .

4.2. Analysis of Simulation Results. The primary goal of bottleneck control is to dissipate the overlong queue; thus, the queue length on the bottleneck link should be one of the important indicators used to assess the control. The average delay across all approaches and the outputs of the two intersections can also be used as assessment indicators, because the splits and capacities of the other approaches will fall and these two indicators will change after a bottleneck control has been adopted.

In [8], the authors found that variations in vehicle lengths were not critical for estimating the blocking occupancy (the absolute error is less than $2 \%$ ), and thus a constant value can be assumed in practice. Meanwhile, the average starting wave speed was found to be approximately $-19.6 \mathrm{~km} / \mathrm{h}$ over 28 cycles, according to field survey data described in $[19,20]$. Therefore, to determine $o_{i, \max }, L_{\text {eff }}$ and $u_{w}$ are assumed to be equal to $10 \mathrm{~m}$ and $-5.28 \mathrm{~m} / \mathrm{s}$, respectively, in this paper.

Based on the simulation environment and parameters, the signal timing plans of the two intersections may be set using the VAP module of VISSIM, and we adjust the data output interval to be equal to the cycle time. These traffic data, including occupancy, maximum queue length, vehicle count,

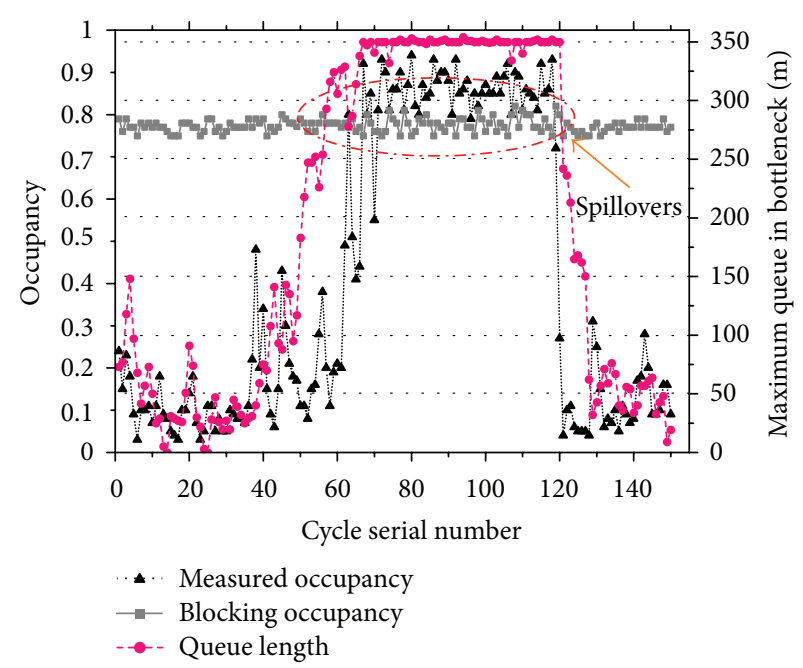

FIGURE 3: Time series of measured and blocking occupancy.

and average vehicular delay, can be obtained by analyzing the output data.

Figure 3 illustrates the following three factors: (1) the block occupancy measured by the vehicle count at the fixed detector in each cycle; (2) occupancy with the cycle time used as the rolling step before the bottleneck control is implemented; (3) the measured maximum queue length of $L_{i+1,3}$ in each cycle before the bottleneck control is implemented.

In addition, we can observe that nearly all of the measured occupancy is greater than the block occupancy from the 70th to the 120th signal cycle, and the corresponding average queue length during the interval is close to the link length of the bottleneck, which indicates the appearance of spillovers. Meanwhile, the measured occupancy and average queue length decrease intensely after the 121st signal cycle, which indicates the dissipation of the spillover.

Figure 4(a) illustrates the maximum queue length in the bottleneck link before and after the bottleneck control is implemented, and shows that the difference between the two control modes is very little when the traffic flow is low. The bottleneck control would be adopted when the maximum queue length is equal to the bottleneck link length, and the maximum queue length should then fall as the inputs decrease and the outputs increase. The maximum queue length cannot decrease to the lowest permissible value ( $150 \mathrm{~m}$ in this paper) in $400 \mathrm{~s}$ (the time allocated to the control), because the residual queue at the upper intersection is underestimated when determining $\Delta S_{a}$. Meanwhile, the maximum and average decrements of the maximum queue length per cycle before and after bottleneck control during the peak hour are about $104 \mathrm{~m}$ and $36.1 \mathrm{~m}$, respectively, which indicate that the congestion state at the bottleneck link is alleviated to some extent.

Vehicles discharge at approximately the saturated flow rate, $Q$ (in vehicles per second), on all approaches during peak hours, and the system outputs (in vehicles per cycle) only depend on the lost time per cycle; meanwhile, the outputs of the system are only determined by the traffic flow during 


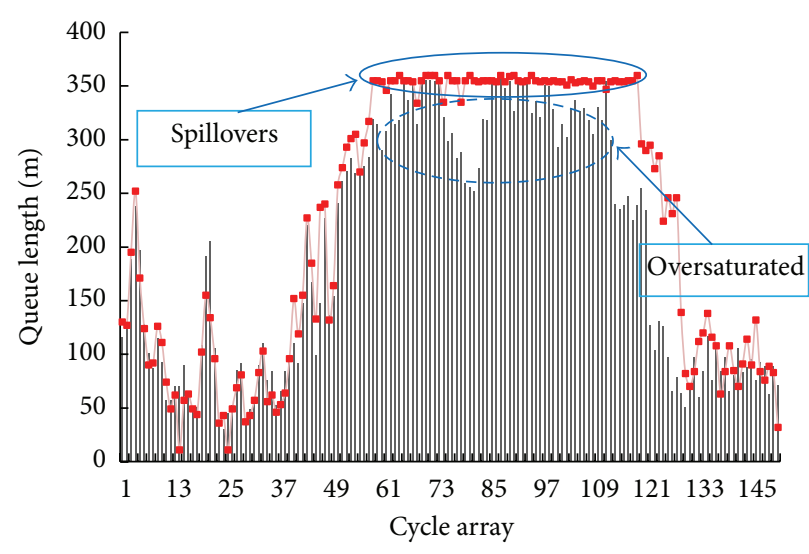

Maximum queue length with bottleneck control -- Maximum queue length without bottleneck control

(a)

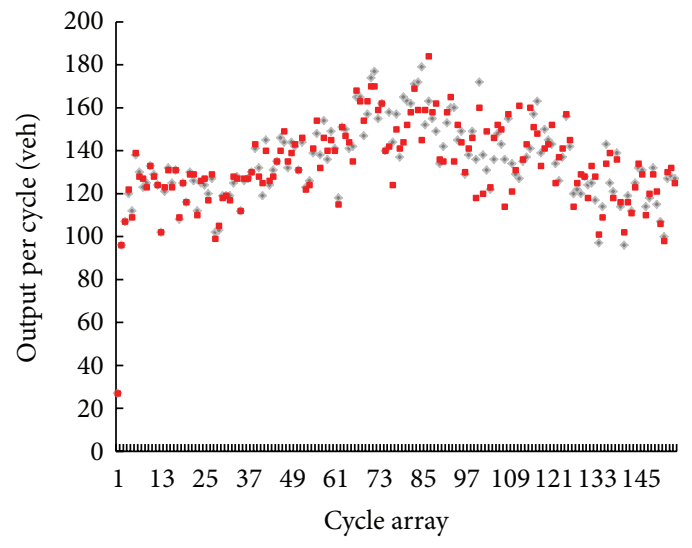

- Output per cycle after bottleneck control

- Output per cycle before bottleneck control

(b)

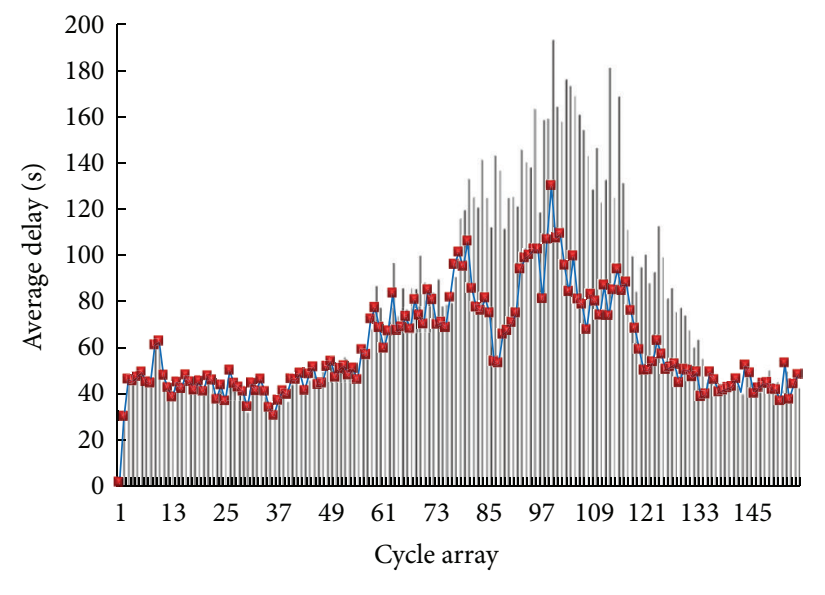

$\square$ Average delay before bottleneck control

- - Average delay after bottleneck control

(c)

FIGURE 4: Changes in indicators before and after implementing the bottleneck control: (a) maximum queue length; (b) outputs per cycle; (c) average vehicular delay.

off-peak hours. Thus, the outputs are similar before and after the bottleneck control if the lost time and traffic flow are kept invariant (the maximum and average variations of the output per cycle before and after bottleneck control during the $5 \mathrm{~h}$ simulation period are 34 veh and 5.18 veh, resp.), which can be seen in Figure 4(b).

Figure 4(c) shows the average vehicular delay per cycle before and after the bottleneck control adopted. It is clear that, when the traffic flow increases above a specific threshold, the performance levels of all approaches at the upstream intersection decrease drastically, because fewer vehicles are able to pass through due to the influence of spillovers. However, the number of spillovers will decline after a bottleneck control has been adopted, and the vehicles will discharge normally on $L_{i, 2}$ and $L_{i, 4}$, making the average delay during rush hours after the bottleneck control has been implemented less than it was before implementation. In Figure 4(c), the maximum and average decrements of the average vehicular delay per cycle before and after bottleneck control during the peak hour are about $89.6 \mathrm{~s}$ and $27.61 \mathrm{~s}$, respectively, and we can draw the conclusion that the operational efficiency of upstream and downstream intersections are improved significantly.

\section{Conclusions}

We have presented a methodology for estimating the average occupancy per cycle using traffic data collected by fixed detectors and determined the threshold of occupancy that indicates a spillover based on shock wave theory. The capacity adjustments for upstream inputs and downstream outputs to and from the bottleneck link were determined within a fixed optimization interval, and the split increase of the input stream and the split decrease in the output stream are defined taking the splits in the original control scheme as weights. This paper has also proposed an optimization method for those phases with no relation to the bottleneck link. 
As traffic flow in an urban street network is unrepeatable, we have to collect traffic data and evaluate the effect of the new methods using a VISSIM simulation, using average vehicular delay, queue length on the bottleneck link and outputs per cycle from the two intersections as the assessment indicators. The results show that bottleneck control can effectively reduce the frequency of spillovers and may also lower the queue length on the bottleneck link. The average vehicular delay is similar before and after the bottleneck control during offpeak hours, but reduces considerably during peak hours, which indicates that the efficiency of the two intersections could be increased by adopting a bottleneck control. The outputs of one approach are equal to the traffic flow at offpeak hours, and the discharging rate for all approaches is close to the saturated flow during peak hours, which may explain why the outputs per cycle are very similar before and after implementing the bottleneck control.

\section{Acknowledgment}

This work was supported by a Grant from the National High Technology Research and Development Program of China (no. 2011AA110304).

\section{References}

[1] A. Skabardonis and N. Geroliminis, "Real-time monitoring and control on signalized arterials," Journal of Intelligent Transportation Systems: Technology, Planning, and Operations, vol. 12, no. 2, pp. 64-74, 2008.

[2] X. K. Wu and H. X. Liu, "A shockwave profile model for traffic flow on congested urban arterials," Transportation Research C, vol. 18, no. 4, pp. 626-638, 2010.

[3] C. Strong and Z. Ye, "Spillover effects of yield-to-pedestrian channelizing devices," Safety Science, vol. 48, no. 3, pp. 342-347, 2010.

[4] J. Chang and B. Bertoli, "New signal control optimization policy for oversaturated arterial systems," in Proceedings of the Transportation Research Board Annual Meeting, Washington, DC, USA, 2010.

[5] X. Wu, H. X. Liu, and D. Gettman, "Identification of oversaturated intersections using high-resolution traffic signal data," Transportation Research C, vol. 18, no. 4, pp. 626-638, 2010.

[6] X. Wu, H. X. Liu, and N. Geroliminis, "An empirical analysis on the arterial fundamental diagram," Transportation Research $B$, vol. 45, no. 1, pp. 255-266, 2011.

[7] N. Geroliminis and A. Skabardonis, "Identification and analysis of queue spillovers in city street networks," IEEE Transactions on Intelligent Transportation Systems, vol. 12, no. 4, pp. 1107-1115, 2011.

[8] N. Geroliminis and A. Skabardonis, "Queue spillovers in city street networks with signal-controlled intersections," in Proceedings of the Transportation Research Board Annual Meeting, Washington, DC, USA, 2010.

[9] D. Quinn, "A review of queue management strategies," Traffic Engineering and Control, vol. 33, no. 11, pp. 600-605, 1992.

[10] D. C. Gazis, "Optimum control of a system of oversaturated intersections," Operation Research, vol. 12, pp. 815-831, 1964.
[11] M. Girianna and R. F. Benekohal, "Dynamic signal coordination for networks with oversaturated intersections," Transportation Research Record, no. 1811, pp. 122-130, 2002.

[12] M. Girianna and R. F. Benekohal, "Signal coordination for a two-way street network with oversaturation Intersections," in Proceedings of the Transportation Research Board Annual Meeting, Washington, DC, USA, 2003.

[13] B. Park, C. J. Messer, and T. Urbanik, "Traffic signal optimization program for oversaturated conditions: genetic algorithm approach," Transportation Research Record, vol. 1683, pp. 133142, 1999.

[14] B. Park, C. J. Messer, and T. Urbanik, "Enhanced genetic algorithm for signal-timing optimization of oversaturated intersections," Transportation Research Record, vol. 1727, pp. 32-41, 2000.

[15] G. F. List and M. Cetin, "Modeling traffic signal control using Petri nets," IEEE Transactions on Intelligent Transportation Systems, vol. 5, no. 3, pp. 177-187, 2004.

[16] R. W. Denney, L. Head, and K. Spencer, "Signal timing under saturated conditions," Tech. Rep. FHWA-HOP-09-008, U.S. Department of Transportation, Federal Highway Administration, Washington, DC, USA, 2008.

[17] A. G. Sim and K. W. Dobinson, "The sydney coordinated adaptive traffic (SCAT) system philosophy and benefits," IEEE Transactions on Vehicular Technology, vol. VT-29, no. 2, pp. 130137, 1980.

[18] P. Mirchandani and L. Head, "A real-time traffic signal control system: architecture, algorithms, and analysis," Transportation Research C, vol. 9, no. 6, pp. 415-432, 2001.

[19] Z. W. Qu, D. H. Wang, and R. H. Yao, "Kinematic model of start wave at signalized intersection," Journal of Jilin University (Engineering and Technology Edition), vol. 38, no. 2, pp. 268-272, 2008 (Chinese).

[20] R. H. Yao, "Sensitivity analysis of optimization models for isolated intersections with short left-turn lanes on approaches," Journal of Advanced Transportation, vol. 47, no. 1, pp. 28-42, 2013. 


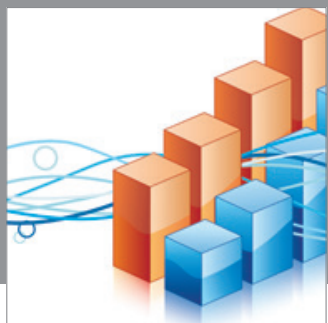

Advances in

Operations Research

mansans

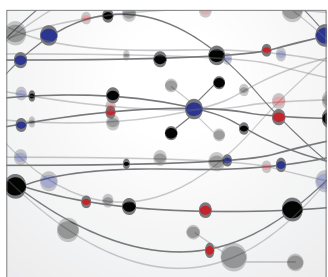

The Scientific World Journal
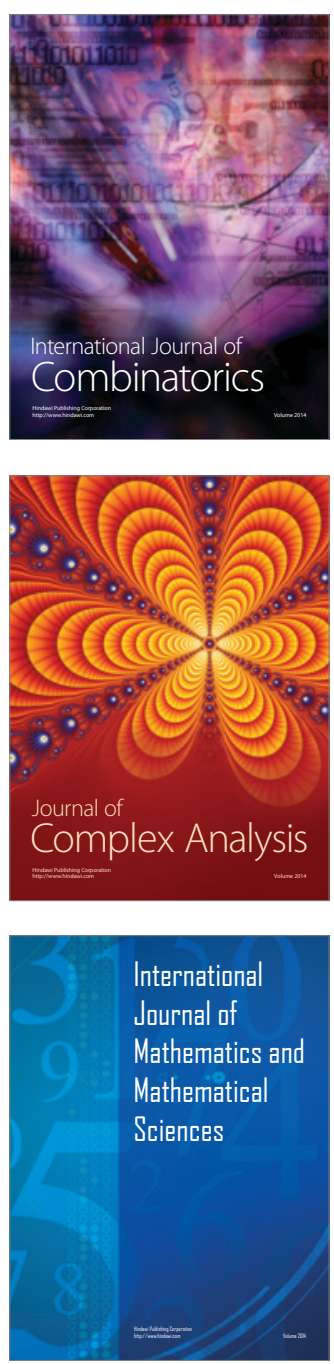
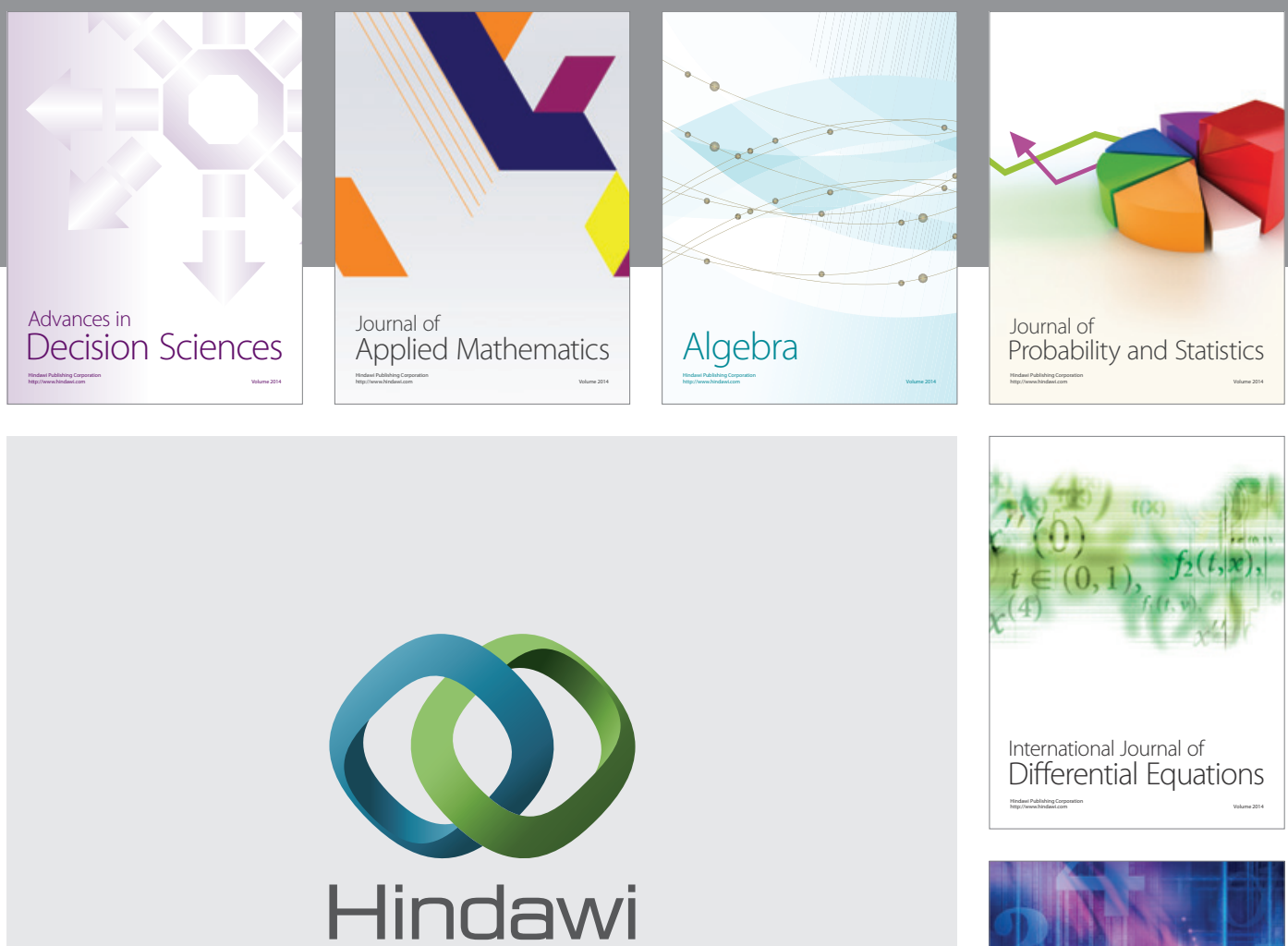

Submit your manuscripts at http://www.hindawi.com
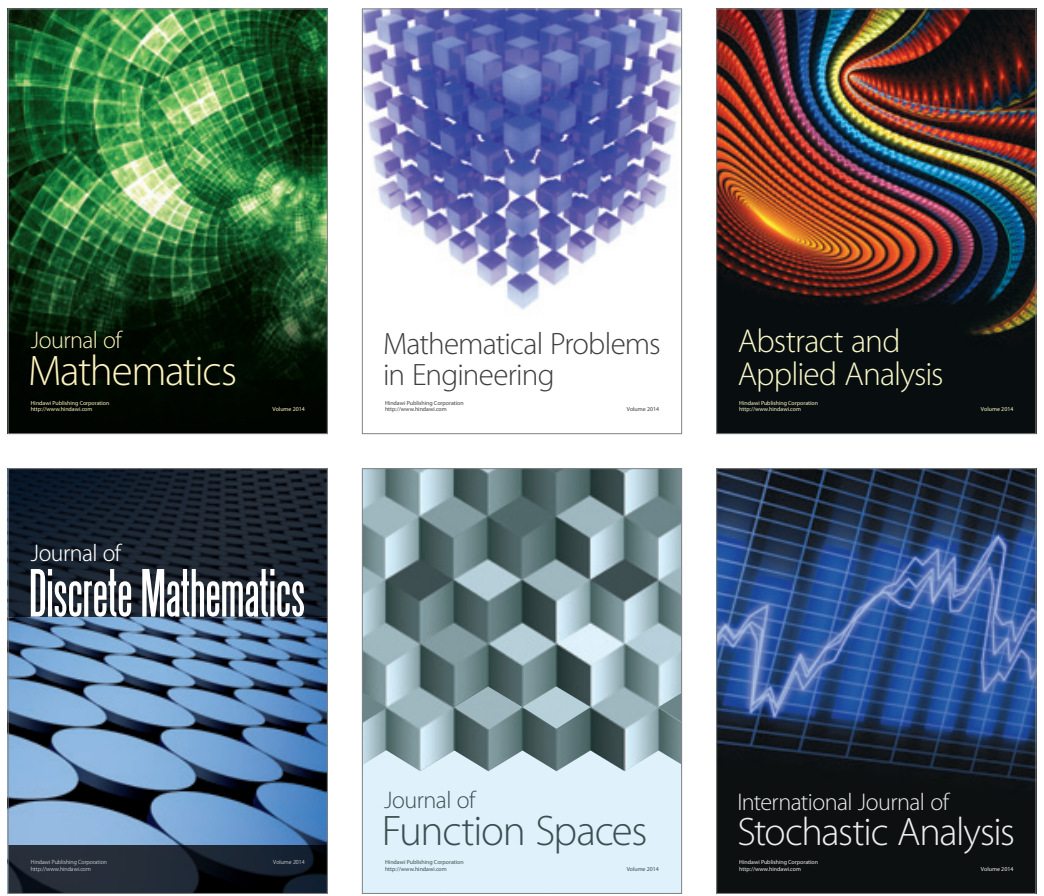

Journal of

Function Spaces

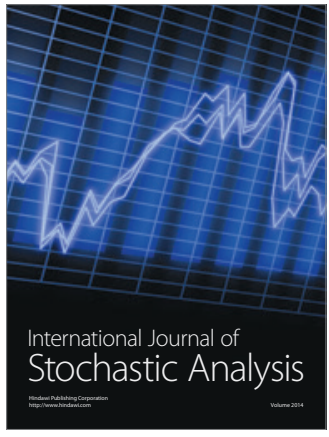

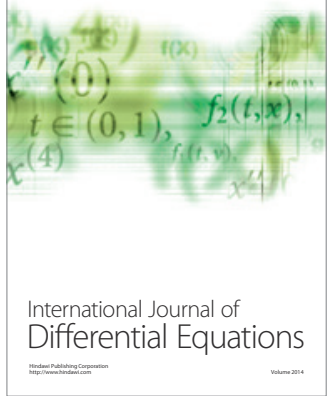
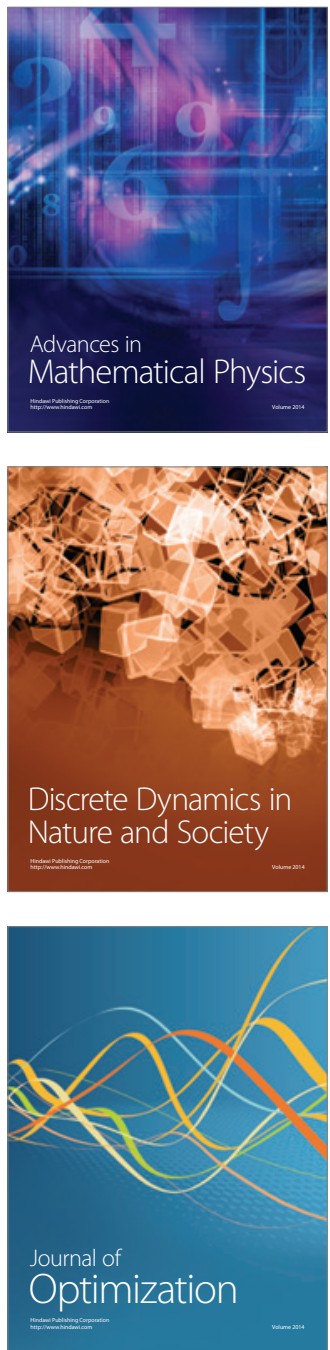